\title{
Oncogenomics of the Hormone-responsive Breast Cancer Phenotype by NGS
}

\author{
Alessandro Weisz \\ Laboratory of Molecular Medicine and Genomics, Faculty of Medicine and Surgery, Molecular Pathology and Medical \\ Genomics Unit, S. Giovanni di Dio e Ruggi d'Aragona University Hospital, University of Salerno, Salerno, Italy \\ http://www.labmedmolge.unisa.it/
}

Breast cancer (BC) comprises an heterogeneous group of diseases characterized by different biological history, clinical phenotype and responsiveness to therapy. Among the factors that contribute substantially to breast carcinogenesis and tumor progression, ovarian hormones - in particular estrogen - have long been known to play a pivotal role. In the mammary gland these steroid hormones control differentiation and growth via two intracellular receptors, ER $\alpha$ and $\beta$, which are ligand-dependent transcription factors belonging to the nuclear receptor family of transcriptional regulators. Upon hormone binding, ERs bind to multiple sites in chromatin of BC cells and thereby act at gene and epigene level to exert a direct control on specific genetic networks driving proliferation, survival and differentiation status of the cell. In breast tumors, elevated levels of ER $\alpha$ and reduced levels of ER $\beta$ are observed from the early stages of the disease, suggesting a dual role for these regulatory factors in breast cancer initiation and progression, with ER $\alpha$ exerting an oncogenic role and ER $\beta$ oncossuppressive functions. Specific gene expression patterns mark the clinical and pathological status of BC lesions and its responsiveness to pharmacological treatments, including hormone therapy with anti-estrogens. The functional relationships between the two ER subtypes and genes characterizing the different clinical phenotypes of breast cancer are not known and are the main focus of our research. To this end, our laboratory is implementing several genome-wide analytical approaches based on the use of NGS and microarrays to investigate ER actions and estrogen signalling in cell models and primary BCs. These studies include: (a) identification of ER and their coregulatory factors interaction with chromatin and mapping of histone marks and other epigenetics codes by ChIPSeq; (b) quantitative analyses of mono- and multi-allelic CpG island methylation by high-throughput DNA methylation microarrays and NGS (methylated DNA IPP sequencing MeDIP-Seq and MBD-Seq, bisulfite-based MethylC-Seq and RRB-Seq); (c) miRNA and other small RNA profiling by miRNA-Seq; (d) ribonucleoprotein-associated RNA identification by RIP-Seq; (e) genome wide search for gene mutations (transitions/transversions, indels, etc) in primary BCs by exome sequencing.

Interpretation and application to the clinical setting of the results obtained with these global analytical approaches require robust statistical tools and innovative bionformatics analyses, that we are interested to implement also in scientific collaborations to be established within SEQAHEAD. 